\title{
Steel Bars Identification in Reinforced Concrete Structures by Using ANN And Magnetic Fields
}

\author{
N. P. de Alcantara Jr. and M. E. L. Gasparini \\ São Paulo State University, Brazil
}

\begin{abstract}
This work proposes a methodology for non destructive testing (NDT) of reinforced concrete structures, using superficial magnetic fields and artificial neural networks, in order to identify the size and position of steel bars, embedded into the concrete. For the purposes of this paper, magnetic induction curves were obtained by using a finite element program. Perceptron Multilayered (PML) ANNs, with Levemberg-Marquardt training algorithm were used. The results presented very good agreement with the expect ones, encouraging the development of real systems based upon the proposed methodology.
\end{abstract}

\section{Introduction}

The inspection of the constitutive elements (steel bars) of the armature of reinforced concrete is a particularly hard problem in Non Destructive Test (NDT) area, because. these elements are completely immersed in an opaque solid media, the concrete. In the past two decades several NDT techniques have been used in the evaluation of the general conditions of reinforced concrete. These techniques relies on elastic waves based techniques [1], and electromagnetic waves based techniques. Electromagnetic waves based techniques can be divided into techniques based on magnetostatic fields [2], induced eddy currents [3], ground penetrating radar (GPR) [4], and X-ray techniques [5]. Each one of these techniques has advantages, disadvantages as well as limitations. Techniques based upon elastic waves present troubles when used to detect anomalies or the presence of objects of small dimensions, due to the large wavelength of this kind of wave. Techniques based upon high frequency electromagnetic waves present problems due to the magnetic properties of the concrete, that present interference effects in this frequency range. Finally, the techniques based on ionizing radiation (X-ray and gamma rays) present serious risks to the health of workers and to the environment.

This work proposes a new methodology for the inspection of steel bar immersed in concrete structures, based in the analysis of magnetic induction curves, created at the region of the structure by electromagnetic devices specially designed for this purpose. The analysis of the magnetic induction curves is done by means of artificial neural networks. For the purpose of this paper, the magnetic inductions values were simulated using a finite element program [6].

\section{The Proposed Methodology}

The methodology proposed in this paper for the localization and identification of steel bars in reinforced concrete structures consists in the utilization of artificial neural networks for pattern classification of electromagnetic signatures presented by the steel bars embedded into the concrete, at the concrete surface, when static and/or quasi-static electromagnetic fields are generated in the region of the concrete structure. The presence of the steel bars within the concrete will slightly disturb the field distribution at the concrete surface. This field perturbation will depend of the size, position and number of bars within the concrete. Each bars configuration will produce an unique deviation curve, called magnetic signature of the bar (or bars). When a large number of samples are taken, artificial neural networks can be used to create an input/output relationship between the magnetic signature and the bar (or bars) configuration.

In order to obtain a better comprehension of the proposed methodology, it will used to identify a steel bar embedded in the concrete. First of all, hundreds of curves of induction magnetic deviations must be obtained. For the purpose of this paper, they were generated using a finite element program [6]. The curves represent variations of the position and size of the bar into the concrete. Figure (1a) illustrate the variations of the position of the bar in the horizontal axis, and figure $1 \mathrm{~b}$ illustrate the variations in the vertical axis. They represent 5 variations in the horizontal position (stepped by $2.5 \mathrm{~mm}$ ) and 30 variations in the vertical position (stepped by $2 \mathrm{~mm}$ ). By this way, 150 variations in the position were considered. Beyond this, 7 sizes were considered for 

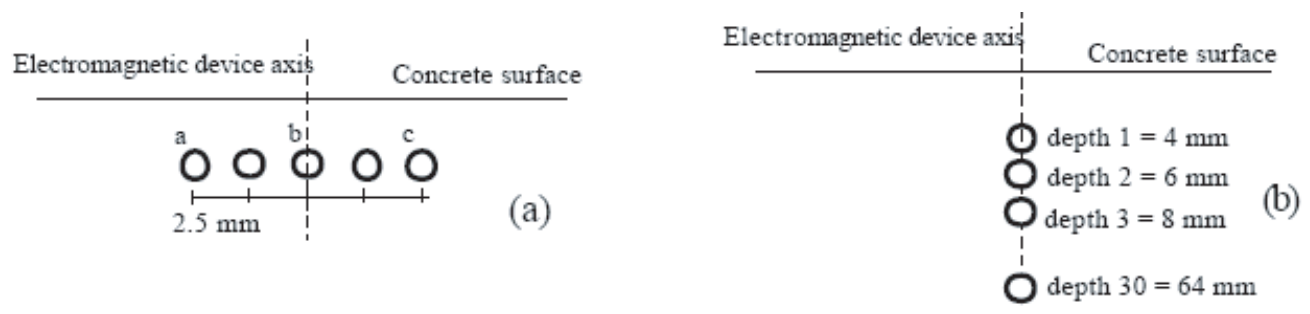

Figure 1: Representation of the variations of the position in the (a) horizontal and (b) vertical direction

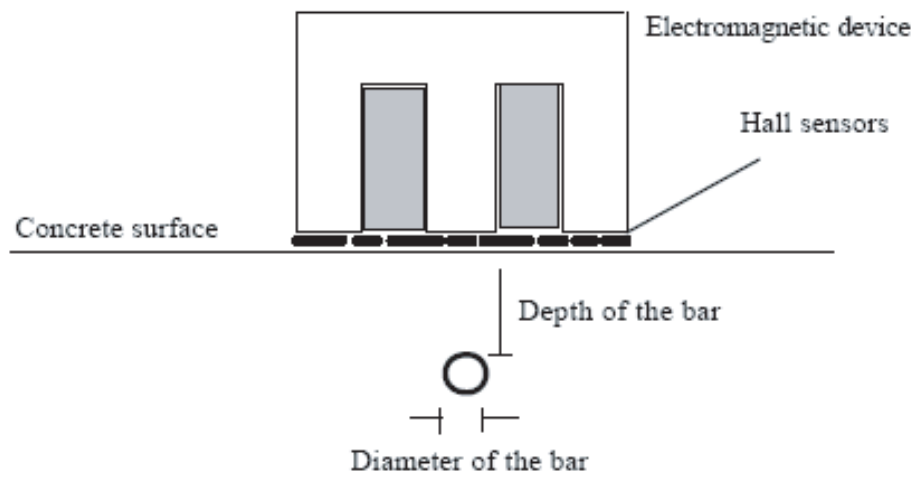

Figure 2: Electromagnetic device idealized for the generation of electromagnetic field

the bar diameter: $8 \mathrm{~mm}, 10 \mathrm{~mm}, 12 \mathrm{~mm}, 14 \mathrm{~mm}, 16 \mathrm{~mm}, 18 \mathrm{~mm}$ and $20 \mathrm{~mm}$. So, 1050 simulations were done with the program.

The magnetic curves are taken at the external surface of the concrete structure. Figure (2) illustrate in a simplified manner a system that can be build in order to obtain the magnetic curves. It must be constituted of a magnetic circuit, a field coil to generate strong electromagnetic field in the region of the bar, and hall sensors, to detect the magnetic induction.

Figures (3) and (4) show samples of magnetic induction deviation curves at the depths of 10 and $30 \mathrm{~mm}$, respectively. The presented curves permits to observe the variations in the magnetic induction in function of the position and size of the bar. Lua script language [7] was used to automatize the generation of the curves.

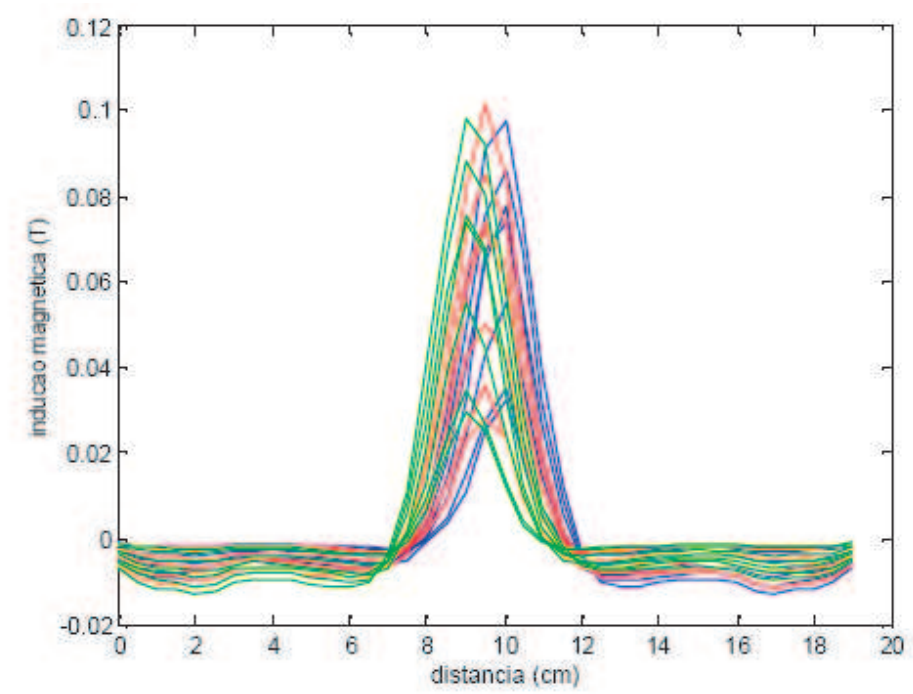

Figure 3: Curves for positions a, b and c (in fig. 3), depth $10 \mathrm{~mm}$. Horizontal axis distance in $\mathrm{cm}$, vertical axis deviation of the normal component of the magnetic induction in $\mathrm{T}$. 


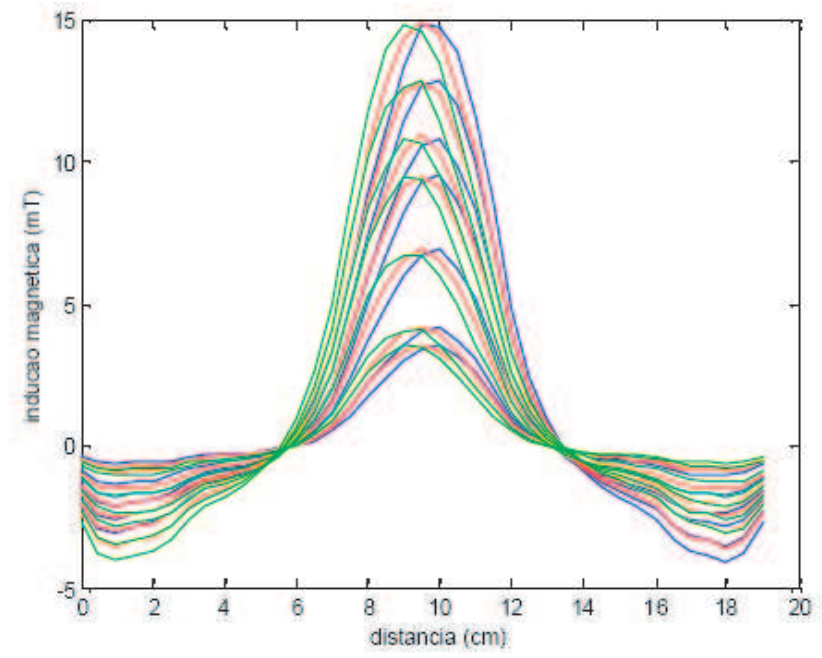

Figure 4: Curves for positions a, b and c (in fig. 3), depth $30 \mathrm{~mm}$. Horizontal axis distance in cm, vertical axis deviation of the normal component of the magnetic induction in $\mathrm{mT}$.

\section{Training of the Neural Networks}

From the 1050 data vectors obtained by the simulations, $60 \%$ of them was used in the training of the neural networks, $20 \%$ was used in the cross validation of the networkss and $20 \%$ was separated for tests with the obtained networks. Perceptron Multilayer (PML) networks, with Levemberg-Marquardt algorithm [8] were used for the training.

The inputs for the networks were vectors containing values of the normal component of the magnetic induction at the surface of the bar. The number of elements in each vector was 41 . None kind of preconditioning was done with the input data.

The desired outputs for the networks were the depth and size of the bar. The output neurons are binary numbers like $(0,0,1)$ for bar diameter equal to $8 \mathrm{~mm},(0,1,0)$ for bar diameter equal to $10 \mathrm{~mm}$ and so on. The depth of the bar was considered in 3 different manners. First, the 150 positions were grouped in 10 groups, with outputs like $(0,0,0,0)$ for depth equal to $6 \mathrm{~mm},(0,0,0,1)$ for depth equal to $12 \mathrm{~mm},(0,0,1,0)$ for depth equal to $18 \mathrm{~mm}$ and so on. As an example, the desired values for the output neurons for a bar with diameter equal to $16 \mathrm{~mm}$ and depth equal to $42 \mathrm{~mm}$ is $(0,1,1,0,1,0,1)$.

Table 1: Obtained results (successfully identification for depth and size) considering 10 possible outputs for the depth.

\begin{tabular}{|c|c|c|c|c|}
\hline Network & Neurons on & \multirow{2}{*}{$\begin{array}{c}\text { Neurons on } \\
\text { hidden layer 1 }\end{array}$} & \multicolumn{2}{|l|}{ Success in the identification (\%) } \\
\cline { 4 - 5 } & hidden layer 2 & depth & size \\
\hline 1 & 10 & 10 & 96.67 & 99.05 \\
\hline 2 & 15 & 10 & 99.52 & 99.52 \\
\hline 3 & 15 & 15 & 100.0 & 99.52 \\
\hline
\end{tabular}

After this, the 150 positions were grouped in 15 groups with outputs like $(0,0,0,0)$ for depth $4 \mathrm{~mm},(0,0,0,1)$ for depth $8 \mathrm{~mm},(0,0,1,0)$ for depth $12 \mathrm{~mm}$ and so on. Finally the 150 position were grouped in 30 groups, with outputs like $(0,0,0,0,0)$ for depth equal to $4 \mathrm{~mm},(0,0,0,0,1)$ for depth equal to $4 \mathrm{~mm}$ and so on.

Table 1 summarizes the results for 3 training sections for the first configuration, table 2 the results for the second configuration and table 3 shows the results for the third configuration.

The presented results agree very well with the expected ones, and confirm that artificial neural networks can be successfully used in the analysis of magnetic signatures of steel bars embedded in reinforced concrete succesfully. 
Table 2: Obtained results (successfully identification for depth and size) considering 15 possible outputs for the depth.

\begin{tabular}{|c|c|c|c|c|}
\hline Network & Neurons on & \multirow{2}{*}{$\begin{array}{c}\text { Neurons on } \\
\text { hidden layer 1 }\end{array}$} & \multicolumn{2}{|l|}{ Success in the identification (\%) } \\
\cline { 4 - 5 } & hidden layer 2 & depth & size \\
\hline 1 & 15 & 15 & 99.05 & 98.52 \\
\hline 2 & 20 & 15 & 99.02 & 98.52 \\
\hline 3 & 20 & 20 & 100.0 & 100.00 \\
\hline
\end{tabular}

Table 3: Obtained results (successfully identification for depth and size) considering 30 possible outputs for the depth.

\begin{tabular}{|c|c|c|c|c|}
\hline \multirow{2}{*}{ Network } & Neurons on & \multirow{2}{*}{$\begin{array}{c}\text { Neurons on } \\
\text { hidden layer 1 }\end{array}$} & \multicolumn{2}{|l|}{ Success in the identification (\%) } \\
\cline { 4 - 5 } & hidden layer 2 & depth & size \\
\hline 1 & 20 & 20 & 69.53 & 96.67 \\
\hline 2 & 25 & 25 & 74.77 & 98.10 \\
\hline 3 & 30 & 30 & 97.62 & 99.04 \\
\hline
\end{tabular}

\section{Conclusion}

This paper presented studies based on computational simulation proposing the use of artificial neural networks in the pattern classification from magnetic induction curves, generated by an electromagnetic device at the surface of reinforced concrete structures, in order to identify the position and the size of the elements of its armature. The obtained results are expressive. The authors believe that the proposed methology can be successfully used with real data achieved in real tests.

\section{REFERENCES}

1. WU, T. T. et al., "On the Study of Elastic Wave Scattering and Rayleigh Wave Velocity Measurement of Concrete with Steel Bar," NDT\&E International, UK, Vol. 33, 401-407, 2000.

2. SCHEEL, H., "Capacity of the Remanent Magnetism Method to Detect Fractures of Steel in Tendons Embedded in Prestressed Concrete," NDT\&E International, UK, Vol. 30, 211-216, 1997.

3. SADEGHI, S. H. H., TOOSI, B., MOINI, R., "On the Suitability of Induction Coils for Crack Detection and Sizing in Metals by the Surface Magnetic Field Measurement Technique," NDT\&E International, UK, Vol. 34, 493-504, 2001.

4. MOLYNEAUX, T. C. K., et al., "Radar Assessment of Structural Concrete Using Neural Networks," NDT\&E International, UK, Vol. 34, 445-456, 2001.

5. MITCHELL, T. M., "Radioactive/Nuclear Methods," CRC Handbook on Non- Destructive Testing of Concrete, USA, CRC Press, 227-252, 1991.

6. MEEKER, D, "Finite Element Magnetic MethodC User Manual,Version 3.3," Germany, 2002.

7. HAYKIN, S., Neural Network: A Comprehensive Foundation, Prentice Hall, USA, 842, 1998, ISBN 013273-350-1.

8. IERUSALIMSCHY, R., DE FIGUEIREDO, L. H. and CELES, W. Lua, "An Extensible Extension Language" Software: Practice \& Experience, Vol. 26, 635-652. 1996. 